\title{
Analysis of relative age effect in muscular strength of adolescent swimmers
}

\author{
Atar Ö. ${ }^{1 \mathrm{ABDE}}$, Özen G. ${ }^{2 \mathrm{ACDE}}$, Koç H. ${ }^{1 \mathrm{ABE}}$ \\ ${ }^{1}$ Department of Coaching Education, Faculty of Sport Sciences, Çanakkale Onsekiz Mart University, \\ Çanakkale, Turkey \\ ${ }^{2}$ Department of Physical Education and Sports Teaching, Faculty of Sport Sciences, Çanakkale Onsekiz Mart \\ University, Çanakkale, Turkey
}

Authors' Contribution: A - Study design; B - Data collection; C - Statistical analysis; D - Manuscript Preparation; E - Funds Collection.

\begin{tabular}{|c|c|}
\hline \multicolumn{2}{|l|}{ Abstract } \\
\hline Purpose: & $\begin{array}{l}\text { The aim of this study is to analyse the effect of relative age in anthropometric measurements, muscular } \\
\text { strength of adolescent swimmers. }\end{array}$ \\
\hline Material: & $\begin{array}{l}\text { The study sample was composed of } 40 \text { volunteers adolescent well-trained swimmers. Participants were } \\
\text { grouped as swimmers born in the first quarter (January, February and March) and in the last quarter } \\
\text { (October, November and December) of the same year. Anthropometric measurements were taken from } \\
\text { each participant, and body mass index of participants were calculated. To determine performance } \\
\text { parameters, handgrip, back and leg strength tests were performed on participants. The level of statistical } \\
\text { significance was set at } p<.05 \text {. }\end{array}$ \\
\hline Results: & $\begin{array}{l}\text { Statistical analysis showed that there were statistically significant differences in the body height and } \\
\text { BMI ( } p<.05) \text {, but body weight differences were not statistically significant between swimmers who are } \\
\text { born first and last quarter of the same year ( } p>.05) \text {. There were significant differences in the means } \\
\text { of absolute leg and handgrip strength between relative age groups. There were statistically significant } \\
\text { differences in the means of relative back, leg and handgrip strength between groups ( } p<.05) \text {. All mean } \\
\text { relative strength values of participants significantly differed in favour of the group born in the first } \\
\text { quarter of the year. }\end{array}$ \\
\hline Conclusions: & $\begin{array}{l}\text { As a result of this study, it is determined that relative age has an effect on the measured anthropometric } \\
\text { and muscular strength parameters of adolescent well-trained swimmers. The findings of this study } \\
\text { revealed that relative age is an important factor to be considered in swimming performance in adolescent } \\
\text { swimmers. }\end{array}$ \\
\hline Key & \\
\hline
\end{tabular}

\section{Introduction}

In many kinds of sports such as basketball, handball, soccer, volleyball, tennis, have been grouped the child and young participants in the competitions according to chronological age. In these sports, child and young athletes who were born on January 1 are grouped with their peers who were born on December 31 of the same year [1, 2]. However, scientific findings have shown that physical growth and biological maturation during childhood and adolescent period can vary even among teenagers of the same age. Grouping according to chronological age in sports provides to produce a competitive environment that may provide advantages for relatively older children and young athletes while disadvantaging other athletes in the same category. Herein, relative age effect (RAE) is considered as a major factor caused this situation [3, 4].

Relative age is calculated based on the month when the individuals were born in the same calendar year. Numerous researchers have indicated that relatively older athletes in the teenagers and childhood period have a higher physical performance than younger peers $[5,6]$. These findings have been revealed that the early maturing teenagers may have potential athletic advantages in terms of body size, strength, endurance, and speed, especially

\footnotetext{
(c) Atar Ö., Özen G., Koç H., 2019

doi:10.15561/18189172.2019.0501
}

in the teenage period [7]. Therefore, relatively older children and young athletes have a lot more opportunity to take part in sports competitions and, consequently, can improve their psychological, technical, and tactical skills, supporting higher athletic performance [8]. In this context, RAE is commonly defined as differences in cognitive or physical performance by chronological age among young athletes who have grouped in the same competition category $[9,10]$.

Adolescence period is the period of most rapid physical growth due to hormonal and metabolic changes in the body. Especially, the most rapid growth occurs in the limbs and muscles. During the rapid growth phase of adolescence, the muscle strength increases and peaks at around 20-25 age [11-13]. Differences in muscle strength depend on age, gender, nutrition, the level of physical activity and muscle functions [14-16]. Age is an important indicator of physical development and muscle strength of children and young people. In this regard, the relative age is to be considered a major parameter in terms of muscle strength development. Existing studies published on this matter have focused on the effect of relative age on the athletic performance of players in team sports such as; basketball, football and hockey. $[1,17]$. Swimming is a sport discipline which muscle strength and body size are highly effective on athletic performance $[12,19]$. Therefore, the RAE 
may have a significant impact on athlete development in swimming. In this regard, the investigation of the effect of relative age on muscular strength in adolescent welltrained swimmers may provide important findings. To this end, the aim of this study was to analysis of RAE in muscular strength of adolescent swimmers.

\section{Materials and Methods}

Subjects

A total of 40 male swimmers who was born out in the first quarter (January, February and March) and in the last quarter (October, November and December) of 2004 participate in this study. The participants did not have a serious disability in the last 6 months and they had regular swimming training for least 5 years. The birth dates of the participants were calculated by taking into account the information on their national identification cards. Both participants and their parents were informed about the purpose and the test protocols of the study. Written informed consent forms were obtained from the parents of the adolescent swimmers who agreed to participate voluntarily in this study. This research was conducted in accordance with the Helsinki Declaration.

\section{Data Collection}

The data for this research was obtained from a standardized data extraction form, anthropometric measurements, and muscular strength tests. The standardized data extraction form was used to record age, training age and self-reported injury history. Anthropometric measurements included body weight and height. Body mass index (BMI) of the participants were calculated using the following formula: body mass $(\mathrm{kg}) /$ height $^{2}(\mathrm{~m})$. Back, leg and handgrip muscle strengths of the participants in the study were measured with a $1 \mathrm{~kg}$ sensitivity Takei digital handgrip and back/ leg dynamometers. No specific advice on dietary habits was given any of the participants during the study period. All anthropometric measurements and performance tests of participants were measured after an overnight fast. All tests for each participant were performed under the same environmental conditions (at air temperature $22^{\circ} \mathrm{C}$ and with $55 \%$ relative humidity) in an indoor performance lab.

Relative Age

Dates of birth in national identity card were used to determine the relative age of the participants. Relative ages of participants were calculated from the months of birth on their cards. Participants were grouped as swimmers born in the first quarter (January, February and March) and in the last quarter (October, November and December) of the same year.

\section{Anthropometric Measurements}

Anthropometric measurements were taken from each swimmer in line with the techniques set forth by the International Society for the Advancement of Kinanthropometry. Body weight of participants was measured them with in light clothes and without shoes by using a digital scale (TANITA, Japan) to the nearest $0.1 \mathrm{~kg}$ and their body height was measured without shoes, standing in the upright position, looking straight forward by using SECA stadiometer (SECA, Germany) device. All measurements were measured by experienced researchers [20].

Performance Tests

Leg strength

All participants were allowed to 5-10 min warm-up and stretching before the strength tests. Leg strength test was carried out using a leg dynamometer (Takei, Japan). They were requested stood on dynamometer table in their knees bent position at approximately 100 degrees and their hands grabbed the dynamometer bar in arm stretched position. For the inactivation of back muscles, the hips were positioned directly over the ankle joints and the back kept straight. In this position, the participant pulled the bar in front of the body upwards as much as possible. The test was repeated three times with a 2 min rest between each trial and recorded the maximum pull out of three trials.

\section{Back Strength}

Participants were similarly positioned as in the leg strength test, but with the legs straight and the back flexed from the hip with fully extended elbows, until the tips of the index fingers reached the patellae. The pull bar of the device was placed in the hands and the chain length was adjusted. The test was performed three times with a $2 \mathrm{~min}$ rest between each trial, and the best score was recorded.

\section{Handgrip Strength}

Handgrip strength test was performed by using a hand dynamometer. This dynamometer was calibrated and adjusted to suit each participant before the test was performed. They were requested seat in a chair without arm support, and with their hips flexed at $90^{\circ}$ and feet resting on the floor. Their elbow of the test arm was flexed to $90^{\circ}$, the forearm in neutral, and the wrist positioned at $15-30^{\circ}$ of extension and $0-15^{\circ}$ of ulnar deviation. They were asked to squeeze the handle with as much force as possible for three seconds. The test was repeated three times with a 2 min rest for each participant. The highest score was recorded and used for statistical analysis.

\section{Statistical Analysis}

Data analysis was performed with the SPSS (V 15.0, SPSS Inc., Chicago, IL, USA) statistical package. Results were presented as mean (M) and standard deviations (SD). A normality analysis was performed by using Kolmogorov Smirnov tests. As variances showed a normal distribution "Independent-samples t-test" were used to determine whether the participants' physical characteristic and muscular strength performances varied according to relative age groups. The significance level was fixed at 0.05 .

\section{Results}

An independent-samples t-test was conducted to compare the physical characteristics for the first and last quarter groups. The comparison of relative age groups was given in Table 1.

According to results of the $t$-test, there were statistically significance differences in the body height $(t(38)=-3.46, \mathrm{p}=.001)$ and BMI $(t(38)=3.32, \mathrm{p}=.002)$ between first and last quarter groups. However, body 
weight differences were not statistically significant $(p>$ .05 ). The mean body height of first quarter group was higher than last quarter group while the mean BMI of last quarter group was higher than the first quarter group.

The results of muscular strength performance tests of relative age groups were compared and presented in Table 2. Independent sample t-test analysis showed that there were statistically significant differences in the means of absolute leg and handgrip strength between groups $(t(38)$ $=2.70, \mathrm{p}=.010 ; t(38)=3.35, \mathrm{p}=.002$ respectively). The means of absolute leg and handgrip strength significantly differed in favour of the group born in the first quarter of the year. However, the means of absolute back muscular strength did not differ statistically between the groups $(\mathrm{t}$ $(38)=1.88, \mathrm{p}=.068)$.

The results of relative muscular strength performance of the groups were compared and presented in Table 3. Independent sample t-test analysis revealed that there were statistically significant differences in the means of relative back, leg and handgrip strength between groups $(t(38)=3.64, \mathrm{p}=.001 ; t(38)=3.76, \mathrm{p}=.001 ; t(38)=$ $3.82, \mathrm{p}=.001$ respectively). All mean relative strength values of participants significantly differed in favour of the group born in the first quarter of the year.

\section{Discussion}

In this research, we investigated the effect of relative age on the handgrip, back and leg muscular strength in adolescent well-trained swimmers who was born in the first and last quarter of the same year. The present study revealed that the RAE brings about significant differences in body height, BMI, relative back strength and both absolute and relative handgrip and leg strength parameters of adolescent swimmers. Our study shown that relative age is an important variable which might make differences in the physical performance of adolescent swimmers due to make differences in some anthropometrics and muscular strength. Previous research reported that children who are born in first months had better cognitive and physical development than their peers who are born in the last months of same year. This means that child and adolescent athletes who are born in the first quarter of the year tend to outperform those who are born in the last quarter of same year.

Table 1. The comparison of physical characteristics between the relative age groups.

\begin{tabular}{lllllll}
\hline Variables & Group & $\mathbf{n}$ & $\mathbf{M}$ & $\mathbf{S D}$ & $\boldsymbol{t}(\mathbf{3 8})$ & $\boldsymbol{p}$ \\
\hline \multirow{2}{*}{ Height $(\mathrm{cm})$} & First Quarter & 20 & 155.8 & 0.25 & \multirow{2}{*}{3.460} & \multirow{2}{*}{$.001^{*}$} \\
& Last Quarter & 20 & 152.9 & 0,26 & & \\
\multirow{3}{*}{ Weight $(\mathrm{kg})$} & First Quarter & 20 & 46.5 & 2.52 & \multirow{2}{*}{0.825} & .414 \\
& Last Quarter & 20 & 47.1 & 1.59 & & \\
\multirow{2}{*}{$\mathrm{BMI}\left(\mathrm{kg} / \mathrm{m}^{2}\right)$} & First Quarter & 20 & 19.2 & 1.11 & \multirow{2}{*}{-3.323} & $.002^{*}$ \\
& Last Quarter & 20 & 20.1 & 0.71 & & \\
\hline
\end{tabular}

Note. ${ }^{*}: p<.05$

Table 2. The comparison of absolute muscular strength performance between the relative age groups.

\begin{tabular}{lllllll}
\hline Variables & Group & $\mathbf{n}$ & $\mathbf{M}$ & $\mathbf{S D}$ & $\boldsymbol{t}(\mathbf{3 8})$ & $\boldsymbol{p}$ \\
\hline \multirow{2}{*}{ Back Stregth $(\mathrm{kg})$} & First Quarter & 20 & 36.20 & 2.63 & \multirow{2}{*}{1.878} & \multirow{2}{*}{.068} \\
& Last Quarter & 20 & 34.86 & 1.80 & & \\
\multirow{2}{*}{ Leg Strength $(\mathrm{kg})$} & First Quarter & 20 & 42.55 & 2.18 & \multirow{2}{*}{2.704} & \multirow{2}{*}{$.010^{*}$} \\
& Last Quarter & 20 & 40.35 & 2.91 & & \\
\multirow{2}{*}{ Hand Grip Strength $(\mathrm{kg})$} & First Quarter & 20 & 16.36 & 1.38 & \multirow{2}{*}{3.352} & \multirow{2}{*}{$.002^{*}$} \\
\hline
\end{tabular}

Note. ${ }^{*}: \mathrm{p}<.05$

Table 3. The comparison of relative muscular strength performance between the relative age groups.

\begin{tabular}{lllllll}
\hline Variables & Group & N & M & SD & $\mathbf{t ( 3 8 )}$ & $\mathbf{p}$ \\
\hline \multirow{2}{*}{ Back Stregth (kg/body mass) } & First Quarter & 20 & 0.78 & 0.04 & \multirow{2}{*}{3.643} & \multirow{2}{*}{$.001^{*}$} \\
& Last Quarter & 20 & 0.74 & 0.03 & & \\
Leg Strength (kg/body mass) & First Quarter & 20 & 0.91 & 0.03 & \multirow{2}{*}{3.759} & $.001^{*}$ \\
& Last Quarter & 20 & 0.86 & 0.06 & & \\
Hand Grip Strength (kg/body & First Quarter & 20 & 0.35 & 0.03 & \multirow{2}{*}{3.818} & $.001^{*}$ \\
mass) & Last Quarter & 20 & 0.32 & 0.02 & & \\
\hline
\end{tabular}

Note. $*: p<.05$ 
Body composition and anthropometric characteristics are considered as important variables affecting performance in many sports. In literature, it is often stated that elite swimmers tend to tall and ecto-mesomorphic body type [21]. The results of our study indicated that adolescent well-trained swimmers who were born in the first quarter significantly taller than their peers who are born in the last quarter of 2004. In this respect, elite adolescent swimmers who are born in the first quarter of year are more likely to perform better performance in swimming competitions.

Comparison of body weight of the adolescent swimmers who are born in first and last quarter shows no statistically significant difference. However, comparison of their BMI values indicated the BMI score of swimmers who are born in the first quarter was significantly lower than swimmers who are born in the last quarter. Previous researchers have reported that smaller body mass could provide an advantage for swimmers because the metabolic cost of horizontal forward motion would in principle increase with mass [21]. Also, they suggested that BMI was associated with athletic performance and physical fitness components in young swimmers [22]. In this respect, this result indicates that adolescent swimmers who are born in the first quarter may be more advantageous in terms of the body structure in swimming.

Previous study reported that individual strength or power activities including both individual sports, such as tennis, skiing swimming, wrestling, taekwondo and team sports, such as rugby, soccer, and hockey have shown to be particularly susceptible to RAE [2, 5, 23, 24]. The present study shown that there were statistically significant differences in the absolute leg and handgrip strength parameters and relative back, leg and handgrip strength parameters between swimmers who were born first and last quarter of the same year. These differences were in favour of swimmers who were born in the first quarter. These results indicate the greater muscle growth in adolescent swimmers who are born in the first quarter. Muscular strength/power plays an important role in the swimmer to achieve swimming performance success. Moreover, arm, leg and back muscular strength are considered as important determinants of swimming performance [19, 21]. Therefore, adolescent swimmers who are born in the first quarter may be more advantageous in terms of physical performance in swimming competitions.

\section{Conclusion}

As a result of this study, it is determined the relative age have been effects on the measured anthropometric and muscular strength parameters of adolescent welltrained swimmers. Athletic performance in swimming is influenced by many factors such as; physical capacity, age (year), training level, motivation, health, genetic, and personal behaviors (physical activity level and eating habits). Additionally, our findings revealed that relative age is an important factor to be considered in swimming performance in adolescent swimmers. Therefore, the development and performance differences between adolescent swimmers who are born first quarter of the same year and their peers who are born last quarter of the same year should be taken into consideration by trainers and sports experts in swimming events and training. Future research should examine not only the effect of relative age on the physical parameters of swimmers but also their swimming performance in different swimming distance and style.

\section{Acknowledgement}

We gratefully acknowledge the help of all the participant who took part in the study.

\section{Conflict of interests}

The authors state that there is no conflict of interest.

\section{References}

1. Musch J, Grondin S. Unequal competition as an impediment to personal development: A review of the relative age effect in sport. Dev Rev. 2001;21(2):147-167. https://doi.org/10.1006/drev.2000.0516

2. Albuquerque MR, Lage GM, Costa VT da, et al. Relative Age Effect in Olympic Taekwondo Athletes. Percept Mot Skills. 2012;114(2):461-468. https://doi.org/10.2466/05.25.PMS.114.2.461-468

3. Cumming SP, Lloyd RS, Oliver JL, Eisenmann JC, Malina RM. Bio-banding in Sport. Strength Cond J.2017;39(2):34-47. https://doi.org/10.1519/SSC.0000000000000281

4. Cobley S, Baker J, Wattie N, McKenna J. Annual age-grouping and athlete development: A meta-analytical review of relative age effects in sport. Sport Med. 2009;39(3):235-256. https://doi.org/10.2165/00007256-200939030-00005

5. Smith KL, Weir PL, Till K, Romann M, Cobley S. Relative Age Effects Across and Within Female Sport Contexts: A Systematic Review and MetaAnalysis. Sport Med. 2018;48(6):1451-1478. https://doi.org/10.1007/s40279-018-0890-8

6. Nakata H, Sakamoto K. Relative age effect in Japanese male athletes. Percept Mot Skills. 2011;113(2):570- 574. https://doi.org/10.2466/05.10.11.PMS.113.5.570-574

7. HelsenWF, Van WinckelJ, WilliamsAM. Therelativeage effect in youth soccer across Europe. J Sports Sci. 2005; 23:629- 636. https://doi.org/10.1080/02640410400021310

8. FukudaDH.Analysis of the relativeage effectineliteyouthjudo athletes. Int J Sports Physiol Perform. 2015;10(8):1048- 1051. https://doi.org/10.1123/ijspp.2014-0463

9. DelormeN,ChalabaevA, RaspaudM.Relativeageisassociated with sport dropout: evidence from youth categories of French basketball. Scand J Med Sci Sports, 2011;21(1):120- 128. https://doi.org/10.1111/j.1600-0838.2009.01060.x

10.Bell JF, Massey AD, Dexter T. Birthdate and ratings of sporting achievement: Analyses of physical education GCSE results. European Journal of Physical Education, 1997;2:60- 166. https://doi.org/10.1080/1740898970020203

11. Brown KA, Patel DR, Darmawan D. Participation in sports in relation to adolescent growth and development. Transl Pediatr. 2017;6(3):150- 159. https://doi.org/10.21037/tp.2017.04.03

12.Doroshenko EY, Svatyev AV, Iermakov SS, Jagiello W. The use of cardio training facilities in training 7-9-year-old 
judo athletes. Archives of Budo Science of Martial Arts and Extreme Sports. 2017;13:165-72.

13.Romanenko V, Podrigalo L, Iermakov S, Rovnaya O, Tolstoplet E, Tropin Y, et al. Functional state of martial arts athletes during implementation process of controlled activity comparative analysis. Physical Activity Review. 2018;6:87-93. https://doi.org/10.16926/par.2018.06.12

14.ChulaniVL, GordonLP.Adolescent Growth and Development. Prim Care - Clin Off Pract. 2014;41(3):465- 487. https://doi.org/10.1016/j.pop.2014.05.002

15.Jagiello W, Jagiello M, Kalina RM, Barczynski BJ, Litwiniuk A, Klimczak J. Properties of body composition of female representatives of the Polish national fencing team - the sabre event. Biology of Sport. 2017;34(4):401-406. https://doi.org/10.5114/biolsport.2017.70526

16.Kriventsova I, Pashkevych S, Iermakov S, Bartik P, Michal J, Nosko M, et al. Fitness - aerobic training of 15 - 17 years' age girl students, who have significant risk of deviations in backbone functional state. Journal of Human Sport and Exercise. 2017;12(4):1289-1297. https://doi.org/10.14198/jhse.2017.124.15

17.Edginton R, Gibson R, Connelly C. Exploring the relative age effect and nation dominance in Olympic Boxing, a review of the last decade. Procedia Engineering. ; 2014;72: 805- 810. https://doi.org/10.1016/j.proeng.2014.06.136

18.Cheung ATH, Ma AWW., Fong SSM, Chung LMY, Bae YH, Liu KPY. Kam KWK, Chung JWY. A comparison of shoulder muscular performance and lean mass between elite and recreational swimmers: Implications for talent identification and development. Medicine (Baltimore). 2018;97(47):1- 5. https://doi.org/10.1097/MD.0000000000013258

19. Aspenes ST, Karlsen T. Exercise-training intervention studies in competitive swimming. Sport Med. 2012;42(6):527- 543. https://doi.org/10.2165/11630760-000000000-00000

20.ISAK; International Society for the Advancement of Kinanthropometry. International Standards of Anthropometric Assessments. Sidney: ISAK; 2001.

21.Lavoie JM, Montpetit RR. Applied Physiology of Swimming. SportMedAnIntJApplMedSciSportExerc. 1986;3(3):165-189. https://doi.org/10.2165/00007256-198603030-00002

22.Strzała M, Tyka A. Physical Endurance, Somatic Indices and Swimming Technique Parameters as Determinants of Front Crawl Swimming Speed at Short Distances in Young Swimmers. Med Sport. 2009;13(2):99-107. https://doi.org/10.2478/v10036-009-0016-3

23.Barnsley RH,ThompsonAH,Barnsley PE. Hockey success and birthdate:therelativeageeffect.CahperJ.1985;45(4):507-512. https://doi.org/10.1177/1012690210371560

24.González-Víllora S, Pastor-Vicedo JC, Cordente D. Relative Age Effect in UEFA Championship Soccer Players. J Hum Kinet. 2015;47:237-248. https://doi.org/10.1515/hukin-2015-0079

\section{Information about the authors:}

Atar Ö.; http://orcid.org/0000-0001-7941-2865; gokmenozen44@gmail.com; Department of Coaching Education, Faculty of Sport Sciences, Çanakkale Onsekiz Mart University; Barbaros, 17100 Kepez/Çanakkale Merkez, Turkey.

Özen G.; (Corresponding author); http://orcid.org/0000-0001-5756-653X; gokmenozen44@gmail.com; Department of Physical Education and Sports Teaching, Faculty of Sport Sciences, Çanakkale Onsekiz Mart University; Barbaros, 17100 Kepez/Çanakkale Merkez, Turkey.

Koç H.; http://orcid.org/0000-0003-2867-9775; gokmenozen44@gmail.com; Department of Coaching Education, Faculty of Sport Sciences, Çanakkale Onsekiz Mart University; Barbaros, 17100 Kepez/Çanakkale Merkez, Turkey

Cite this article as:

Atar Ö, Özen G, Koç H. Analysis of relative age effect in muscular strength of adolescent swimmers. Pedagogics, psychology, medical-biological problems of physical training and sports, 2019;23(5):214-218.

https://doi.org/10.15561/18189172.2019.0501

This is an Open Access article distributed under the terms of the Creative Commons Attribution License, which permits unrestricted use, distribution, and reproduction in any medium, provided the original work is properly cited (http://creativecommons.org/licenses/by/4.0/deed.en).

Received: 19.06 .2019

Accepted: 30.07.2019; Published: 17.09.2019 